\title{
Synergistic anti-tumor effects of dasatinib and dendritic cell vaccine on metastatic breast cancer in a mouse model
}

\author{
NINGNING SONG ${ }^{1 *}$, HULIN GUO $^{2 *}$, JIA REN $^{3}$, SUHONG HAO $^{3}$ and XINCHAO WANG ${ }^{1}$ \\ ${ }^{1}$ Department of Thyroid and Breast Surgery, Fourth Center Clinical College of Tianjin Medical University, \\ Tianjin 300140; ${ }^{2}$ Department of Integrated Traditional Chinese and Western Medicine, The Fifth \\ People's Hospital of Qinghai Province, Xining, Qinghai 810007; ${ }^{3}$ Department of Oncology, \\ Tianjin University of Traditional Chinese Medicine, Tianjin 300193, P.R. China
}

Received January 15, 2016; Accepted July 3, 2017

DOI: $10.3892 / \mathrm{ol} .2018 .8188$

\begin{abstract}
Immunotherapy is currently considered as one of the major anti-tumor modalities, but its efficacy is limited. Dasatinib could improve the expansion and recruitment of cluster of differentiation (CD) $8^{+} \mathrm{T}$ cells and natural killer (NK) cells to the tumor microenvironment. The present study aimed to evaluate the synergistic anti-tumor effects of dasatinib with dendritic cell (DC) vaccine in metastatic breast cancer. Dasatinib with DC vaccine was administered to mice inoculated with 4T1 breast cancer cells. Thereafter, tumor volume was measured every other day. On day 34, lung metastasis was assessed with a stereomicroscope. Tumor proliferation and angiogenesis were determined by immunohistochemistry. Apoptosis in tumor tissues was assessed by terminal deoxynucleotidyl transferase dUTP nick end labeling. The results showed that although there were no significant differences in tumor volumes between the untreated control, DC vaccine and dasatinib groups, the tumor volume was significantly decreased in the combined treatment group compared to the other three groups. Mice in the combined treatment group showed the longest survival time, while mice treated with either single treatment had a slightly increased survival time compared to the untreated control mice. Additionally, the number of metastatic lung nodules was significantly decreased in combined treatment group compared with the dasatinib alone, DC vaccine alone and untreated control groups. Furthermore,
\end{abstract}

Correspondence to: Professor Xinchao Wang, Department of Thyroid and Breast Surgery, Fourth Center Clinical College of Tianjin Medical University, 3 Zhongshan Road, Hebei, Tianjin 300140, P.R. China

E-mail: wangxc010@163.com

${ }^{*}$ Contributed equally

Abbreviations: DC, dendritic cell; MDSC, myeloid-derived suppressor cell; Tregs, regulatory T cell; SFK, SRC family kinase

Key words: breast cancer, dasatinib, dendritic cell, vaccine, lung metastasis the combined treatment group showed significantly reduced intratumoral microvessel density compared to the other three groups. In addition, the ratios of $\mathrm{CD}^{+} \mathrm{T}$ and $\mathrm{NK}$ cells were significantly increased in the combined treatment group compared with the other three groups. These results suggest that dasatinib combined with the DC vaccine is a possible modality for the treatment of metastatic breast cancer.

\section{Introduction}

Breast cancer remains the most common malignant tumor in women worldwide and distant metastases is a notable feature of advanced breast cancer $(1,2)$. Despite recent advances in early diagnosis and multidisciplinary therapeutic management, metastatic breast cancer remains challenging to successfully treat $(3,4)$.

Dasatinib is a potent orally-administered multi-target kinase inhibitor that inhibits several kinases, including breakpoint cluster region protein-Abelson murine leukemia viral oncogene homolog, SRC family kinase (SFK), c-KIT and platelet-derived growth factor receptor- $\beta$, and has been approved for use in patients with imatinib-refractory leukemia $(5,6)$. SFKhasa key role in numerous cellular signaling pathways that not only modulate the behavior of tumor cells, but also regulate immune effector cells (7-11). For example, Christiansson et al (11) found that dasatinib improved the expansion of cluster of differentiation $(\mathrm{CD}) 8^{+} \mathrm{T}$ cells and natural killer $(\mathrm{NK})$ cells in chronic myelogenous leukemia patients. Additionally, patients with $\mathrm{NK} / \mathrm{T}$ cell lineage lymphocytosis tend to exhibit preferable clinical effects compared with patients without lymphocytosis, indicating that the anti-tumor effect is due to activated immune responses (12). However, available phase I and phase II clinical trial data have confirmed only limited benefits from the use of single-agent dasatinib in diverse types of breast cancer, and the overall response rate was only $4.7 \%$ in triple-negative breast cancer (13-16).

Due to the unique ability to initiate and boost powerful anti-tumor $\mathrm{T}$ cell responses, ex vivo-generated dendritic cells (DCs) are essential for immunotherapy to effectively kill the malignant cells. Notably, dasatinib enhanced the therapeutic efficacy of the DC vaccine in vitro and in vivo $(8,17-19)$. Nerreter et al (18) reported that dasatinib enhanced the 
migration of DCs without changing the ability to prime and boost antigen-specific $\mathrm{T}$ cell response by reducing the phosphorylation of inhibitory immune receptor siglec-9 and siglec-3 in vitro using human peripheral blood mononuclear cell-derived DCs. Lowe et al (8) revealed that dasatinib combined with the DC vaccine significantly reduced tumor volume, with enhanced recruitment of $\mathrm{CD}^{+} \mathrm{T}$ cells and DCs to tumor-draining lymph nodes and the tumor microenvironment in M05 melanoma mouse models. However, the combined the effect of dasatinib and DC-based vaccine on advanced breast cancer is unknown.

In the present study, a murine 4T1 orthotopic model was established to mimic human stage IV breast cancer, and the synergistic anti-tumor effects of dasatinib and DC vaccine on metastatic breast cancer were evaluated using this model.

\section{Materials and methods}

Cell culture. The breast cancer 4T1 cell line was obtained from Cell Bank, Shanghai Institutes for Biological Sciences, Chinese Academy of Sciences (Shanghai, China) and cultured in RPMI-1640 Medium (Hy Clone; GE Healthcare Life Sciences, Logan, UT, USA) supplemented with $10 \%$ fetal calf serum (FCS; Tianjin Haoyang Biological Products Technology Co., Ltd., Tianjin, China) at $37^{\circ} \mathrm{C}$.

Mice. A total of $45 \mathrm{Balb} / \mathrm{cmice}$ (32 female and 13 male; 6 weeks old; weight $20 \pm 2$ g) were provided by Animal Center, Chinese Academy of Medical Sciences (Beijing, China) and fed with ad libitum access to purified water and a commercial stock diet which was changed regularly (renewed and added every $12 \mathrm{~h}$ ). All mice were kept in a pathogen-free environment at a temperature of $21 \pm 1^{\circ} \mathrm{C}$ with a $12: 12 \mathrm{~h}$ light: dark cycle and maintained under a relative humidity of $50 \pm 10 \%$. All procedures involving animals were approved by the Ethics Committee of Tianjin Medical University (Tianjin, China).

$4 T 1$ cell lysate. For the preparation of cell lysate, 4T1 cells were washed with PBS three times and the cell concentration was adjusted as $5 \times 10^{7}$ cells/ml. 4T1 cells were lysed by five freeze-thaw cycles accomplished by 15 min liquid nitrogen submersions followed by 5 min water bath incubations at $55^{\circ} \mathrm{C}$. The lysates were centrifuged at $1,800 \mathrm{x} g$ for $15 \mathrm{~min}$ to remove the cell debris at $4^{\circ} \mathrm{C}$. The lysate was aliquoted and stored at $-80^{\circ} \mathrm{C}$ until use.

Tumor lysate-pulsed DCs. Mouse bone marrow-derived cells (BMDCs) were generated from bone marrow precursors. In brief, bone marrow cells were flushed from the femurs and tibias of male BALB/c mice, and subsequently washed and counted. Cells were plated at a concentration of $2 \times 10^{6}$ cells/100-mm Petri dish in $10 \mathrm{ml}$ RPMI-1640 medium supplemented with $10 \%$ FCS, $20 \mathrm{ng} / \mathrm{ml}$ murine recombinant granulocyte-macrophage colony-stimulating factor and $10 \mathrm{ng} / \mathrm{ml}$ murine recombinant interleukin (IL)-4 (PeproTech, Rocky Hill, NJ, USA) in a humidified incubator $\left(5 \% \mathrm{CO}_{2}\right.$ and $37^{\circ} \mathrm{C}$ ). On days 3 and 6 , the media was refreshed. On day 7 , $100 \mu$ cell lysate of 4T1 cells (DC: 4T1, 1:3) was added to the medium and incubated for $18 \mathrm{~h}$. To obtain mature DCs, $1 \mu \mathrm{l} / \mathrm{ml}$ lipopolysaccharide (LPS; Sigma-Aldrich; Merck
KGaA, Darmstadt, Germany) was added to the medium and cultured for $1-2$ days at $37^{\circ} \mathrm{C}$.

Tumor model and treatment protocol. In total, 1x10 4 T1 cells were injected subcutaneously into the right mammary gland of $32 \mathrm{BALB} / \mathrm{cfe}$ male mice on day 0 . On day 10 , the animals were randomized into 4 groups $(n=8)$. To find the optimal dose of dasatinib in improving $\mathrm{CD}^{+} \mathrm{T}$ cell recruitment to the tumor microenvironment, a pre-experiment was performed according to Lowe et al (8) (data not shown). Dasatinib (15 mg/kg; Selleck Chemicals, Houston, TX, USA) was solubilized in $50 \mu \mathrm{l}$ Labrasol [Gattefosse (Shanghai) Trading Co., Ltd., Shanghai, China]. Mice in the dasatinib group were administered with $15 \mathrm{mg} / \mathrm{kg}$ dasatinib by daily oral gavage for 7 consecutive days, beginning on day 10. Mice in the DC vaccine group were injected with $1 \times 10^{6} 4 \mathrm{~T} 1$ lysate-pulsed dendritic cells in $50 \mu 1$ PBS in the right mammary gland surrounding the tumor on days 10 and 17. Mice in the control group were left untreated. The combined treatment group received both dasatinib and DC vaccine, as aforementioned. Tumor size was measured every other day in two dimensions using Vernier calipers, and tumor volume were calculated using the formula, $\mathrm{ab}^{2} / 2$, where $\mathrm{b}$ is the smaller dimension. On day 34 , Mice were sacrificed by cervical dislocation. The primary tumors, lungs, livers and spleen were excised, weighed and breast pulmonary nodules were counted using a stereomicroscope (Nikon, Tokyo, Japan).

Histology and immunohistochemistry. For histological analysis, primary tumor, lung and liver tissue sections were collected and fixed in $10 \%$ formalin for $24 \mathrm{~h}$ at room temperature. Subsequent to being embedded in paraffin, specimens were cut into $5 \mu \mathrm{m}$ sections and were stained with hematoxylin and eosin (H\&E) for $20 \mathrm{~min}$ at room temperature. For immunohistochemistry, the tumor sections were prepared in the same manner as the tissue used for H\&E staining. Following antigen retrieval (10 mmol/lsodium citrate buffer, $\mathrm{pH}$ 6.0; microwave $600 \mathrm{~W}, 10 \mathrm{~min}$ ) all tumor slides were blocked with $5 \%$ bovine serum albumin (Boster Biological Technology, Pleasanton, CA, USA) for $1 \mathrm{~h}$ at room temperature and stained with rabbit anti-mouse natural-killer group 2, member D (NKG2D), CD8, KI67 or CD31 antibodies (cat nos. bs-0938R, bs-10699R, bs-23105R and bs-20322R, respectively; Bioss, Beijing, China) at a dilution of 1:200 at $4^{\circ} \mathrm{C}$ overnight, in accordance with the standard avidin-biotin-peroxidase complex staining procedure. Then, avidin-biotin-peroxidase conjugated goat anti-rabbit IgG (cat no. SA1022; Boster Biological Technology; dilution, ready-to-use) was added for $20 \mathrm{~min}$ at $25^{\circ} \mathrm{C}$. Finally, diaminobenzidine (Boster Biological Technology) was used for staining for 10-30 $\mathrm{min}$ at room temperature. The uniform fields from each section were selected and analyzed by two independent pathologists in a blind manner. Ki67, CD8 and NKG2D-positivecells were counted in five randomly-selected fields from three separate sections at a magnification of x400 under a light microscope (TE200-U; Nikon), with data presented as the percentage of the total number of tumor cells. Microvessel density was counted using five fields per tumor following the criteria described by Weidner et al (20).

Terminal deoxynucleotidyl transferase dUTP nick end labeling (TUNEL). To assess apoptosis, tumor tissues in different 

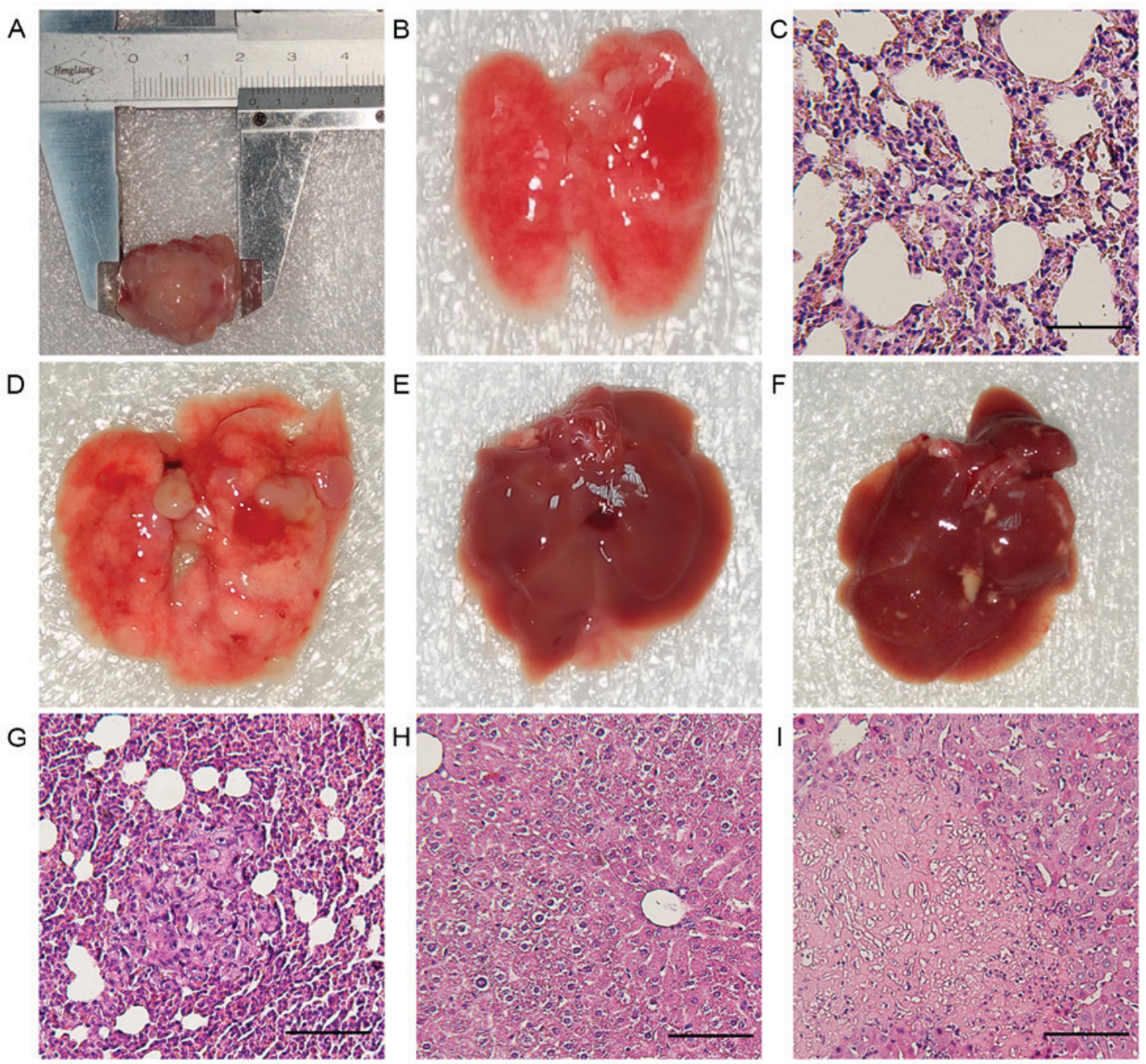

Figure 1. Tumorigenesis and metastasis formation in 4T1 tumor-bearing mice. (A) Primary breast carcinoma extracted from a 4T1 tumor-bearing control mouse. (B and C) Gross and microscopic morphology of lungs extracted from a healthy control mouse. (D) Gross morphology of lungs extracted from a 4T1 tumor-bearing control mouse showing numerous metastatic nodules. (E) Gross morphology of livers extracted from a 4T1 tumor-bearing control mouse. (F) Only one 4T1 tumor-bearing control mouse presented with liver macroscopic yellow necrosis.(G) Microscopic morphology of lungs extracted from a 4T1 tumor-bearing control mouse confirming numerous metastatic nodules. (H) Microscopic morphology of livers extracted from a 4T1 tumor-bearing control mouse. (I) Microscope morphology of livers extracted from a 4T1 tumor-bearing control mouse confirming necrosis. Scale bars, $30 \mu \mathrm{m}$.

groups were subjected to TUNEL assay using the In Situ Cell Death Detection Kit (Roche Diagnostics, Basel, Switzerland), according to the manufacturer's protocol. Cells stained brown were considered positive for apoptosis. At least 10 fields at a magnification of $\mathrm{x} 400$ were randomly selected under a fluorescent microscope, and the total cells were counted in each field. The percentage of apoptotic positive cells was calculated as the number of apoptotic positive cells/total cells.

Statistical analysis. Data were expressed asthemean \pm standard deviation. GraphPad Prism 6.01 (GraphPad Software, Inc., La Jolla, CA, USA) was used for statistical analysis. Statistical significance of differences between two cohorts was analyzed by Student's t test. Statistical significance of differences between three cohorts was analyzed by one-way analysis of variance followed by Student-Newman-Keuls post hoc test. Log-rank (Mantel-Cox) test was used to compare survival curves. $\mathrm{P}<0.05$ was considered to indicate a statistically significant difference.

\section{Results}

Dasatinib and 4T1-antigen-loaded DC vaccine reduced tumor growth in mice synergistically. First tumorigenesis and metastasis formation of $4 \mathrm{~T} 1$ cells were examined in vivo. After 3 weeks, mice developed primary metastatic breast carcinoma (Fig. 1A). The anatomy revealed the presence of numerous macroscopic metastatic foci in all cases. To determine whether the lung nodules were from $4 \mathrm{~T}$ cells, H\&E staining was performed on lung tissues (Fig. 1D and G). In addition, liver tissue was examined by macroscopic and H\&E staining analysis. All livers showed no metastases (Fig. 1E and F), but one liver showed macroscopic necrosis (Fig. 1H and I).

To determine whether the combination regime improved the inhibition of tumor growth, tumor-bearing mice were treated with dasatinib and DC-based vaccine. No significant differences were observed in tumor volumes between the untreated control, DC vaccine and dasatinib groups, but tumor volumes were significantly decreased in the combined 
A

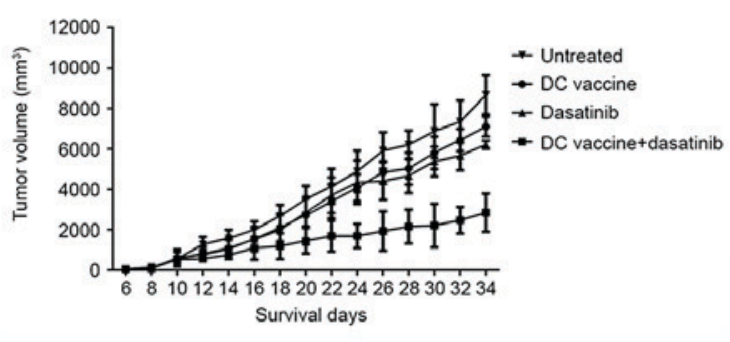

C

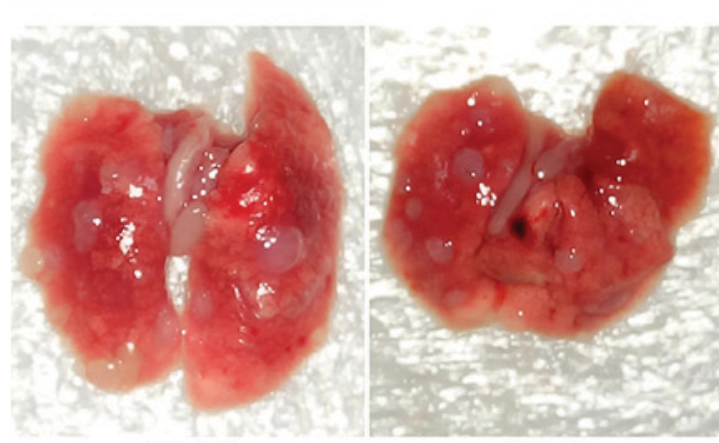

Untreated

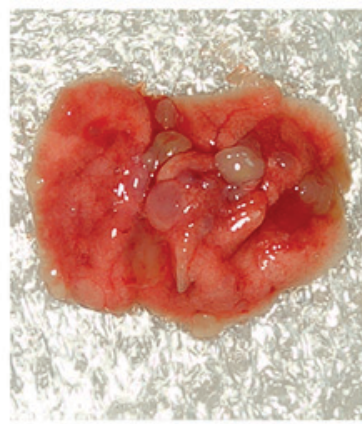

Dasatinib

D

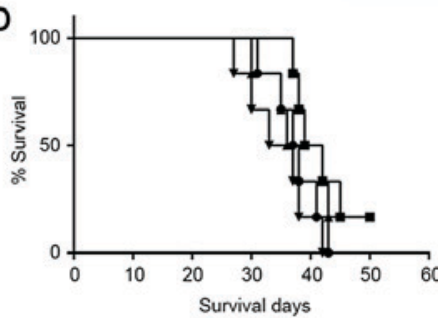

$\mathbf{F}$

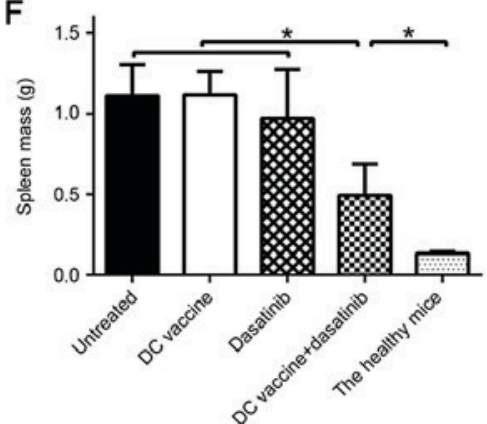

$\mathrm{DC}$ vaccine

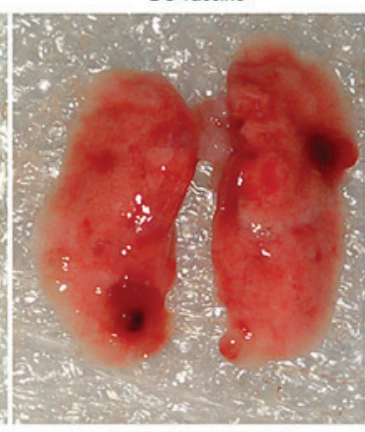

DC vaccine+dasatinib

$\rightarrow$ Untreated

$\rightarrow$ DC vaccine

- Dasatinib

- DC vaccine+dasatinib

E

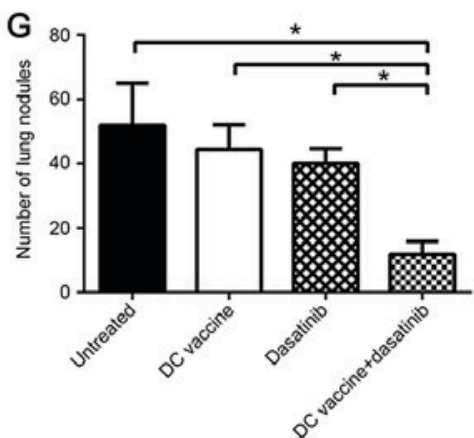

B

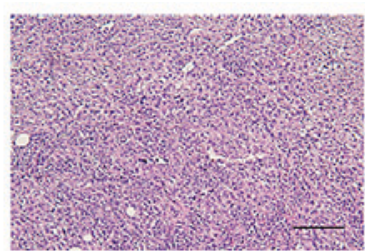

Untreated

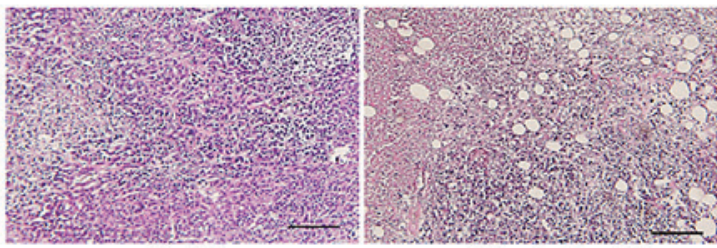

Dasatinib

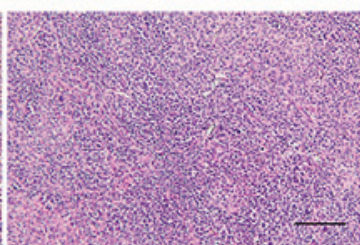

DC vaccine

DC vaccine+dasatinib
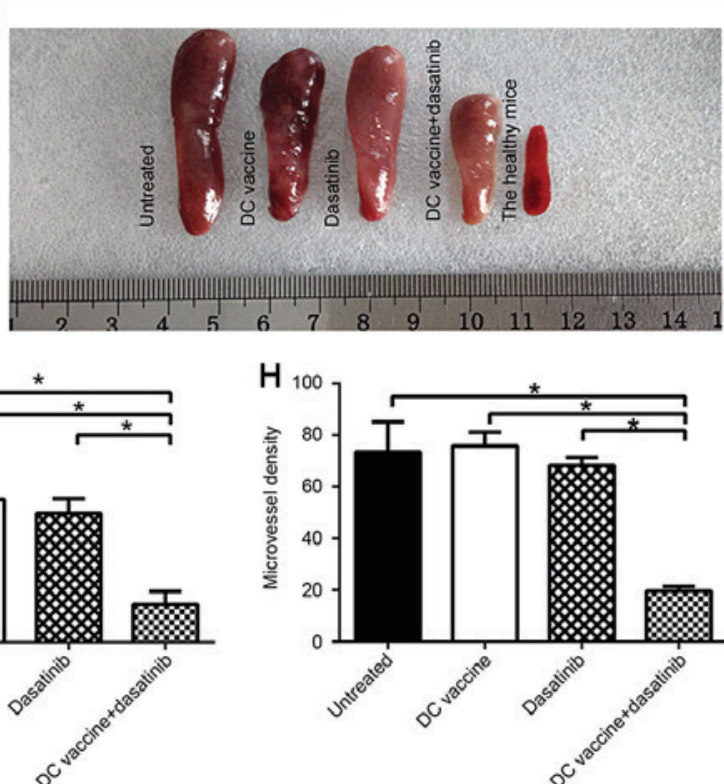

Figure 2. The inhibition of tumor growth, intratumoral angiogenesis and lung metastasis in mice subsequent to different treatments. (A) Tumor volume of the 4T1 breast tumor model was measured every 2 days. Values are presented as the mean \pm standard deviation. (B) H\&E staining of the tumors of each group. Scale bars, $60 \mu \mathrm{m}$. (C) Representative gross lung image of each group. (D) Kaplan-Meier plot showing the survival rate of the indicated group (n=8 per group). (E) Representative spleen images of each group. (F) All spleen were harvested and weighed. (G) Quantification of lung nodules in each group. (H) Microvessel density was assessed using five fields per tumor. "P<0.05. DC, dendritic cell; H\&E, hematoxylin and eosin. 


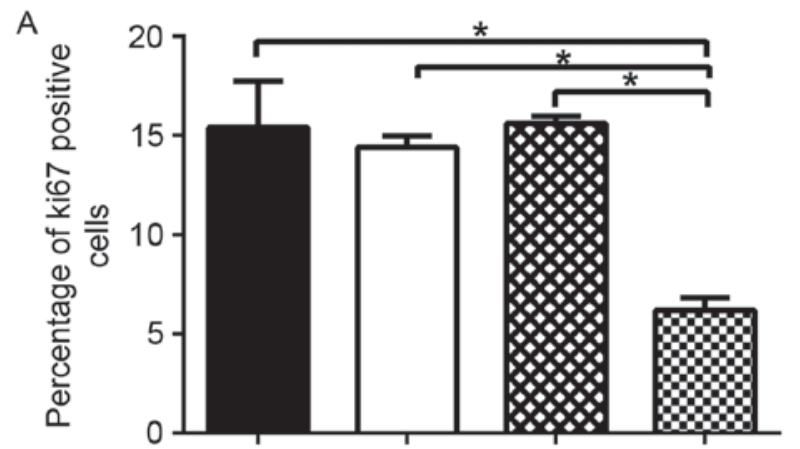

B

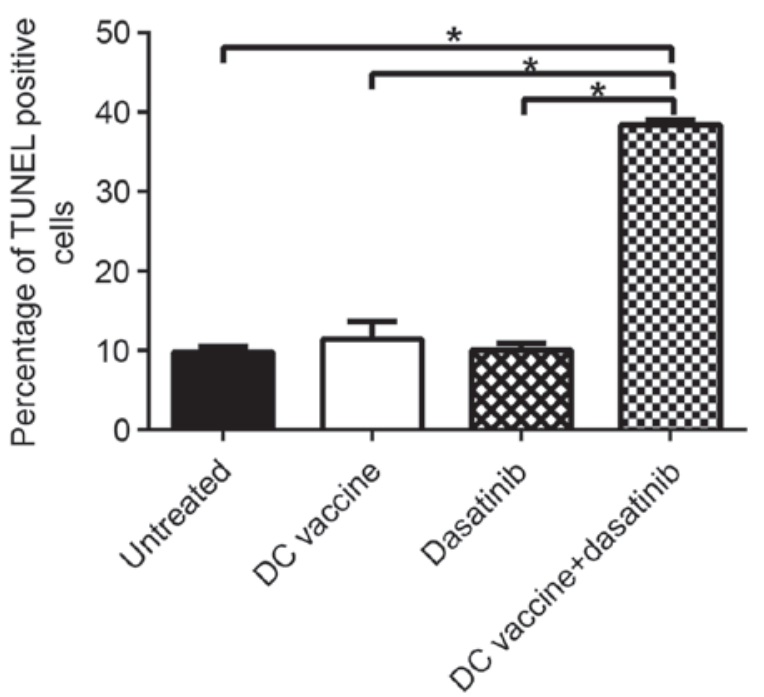

Figure 3. Changes in the proliferation and apoptosis of $4 \mathrm{~T} 1$ tumor cells after different treatments. Immunohistochemical analysis of Ki67 in each group of tumors. (A) Ki67-positive cells in each group of tumors were measured. Immunohistochemical analysis of apoptosis in each cohort of tumors. (B) TUNEL positive cells in each group of tumors were measured. ${ }^{*} \mathrm{P}<0.05$. DC, dendritic cell; TUNEL, terminal deoxynucleotidyl transferase dUTP nick end labeling.

treatment group compared to the other three groups (Fig. 2A; $\mathrm{P}<0.05$ vs. all other groups after $22 \mathrm{~d}$ tumor-inoculation). $\mathrm{H} \& \mathrm{E}$ staining of tumor tissue revealed irregular, disrupted tumor cells with large, blue-black nuclei (Fig. 2B). The spleen were excised and weighed on day 34 (Fig. 2E). The spleen mass was significantly decreased in the combined group or healthy control group compared to other groups (Fig. 2F; $\mathrm{P}<0.05$ ). Mice treated with dasatinib combined with $\mathrm{DC}$ vaccine showed the longest survival time, and mice treated with either single treatment lived slightly longer compared with untreated control mice (non-significant; $\mathrm{P}>0.05$ ); however, these differences were not statistically significant (Fig. 2D).

Combination of dasatinib and DC vaccine elicited superior inhibition on metastatic lung nodules and intratumoral angiogenesis. The mice in all treatment groups were sacrificed prior to lung nodule quantification. The incidence of lung metastases in all four groups was the same, with $100 \%$ of mice developing lung metastases. However, the number of metastatic lung nodules was significantly decreased in the combined treatment group compared with the dasatinib alone, DC vaccine alone and untreated control groups (Fig. 2C and $\mathrm{G} ; \mathrm{P}<0.05)$. Furthermore, immunohistochemistry of
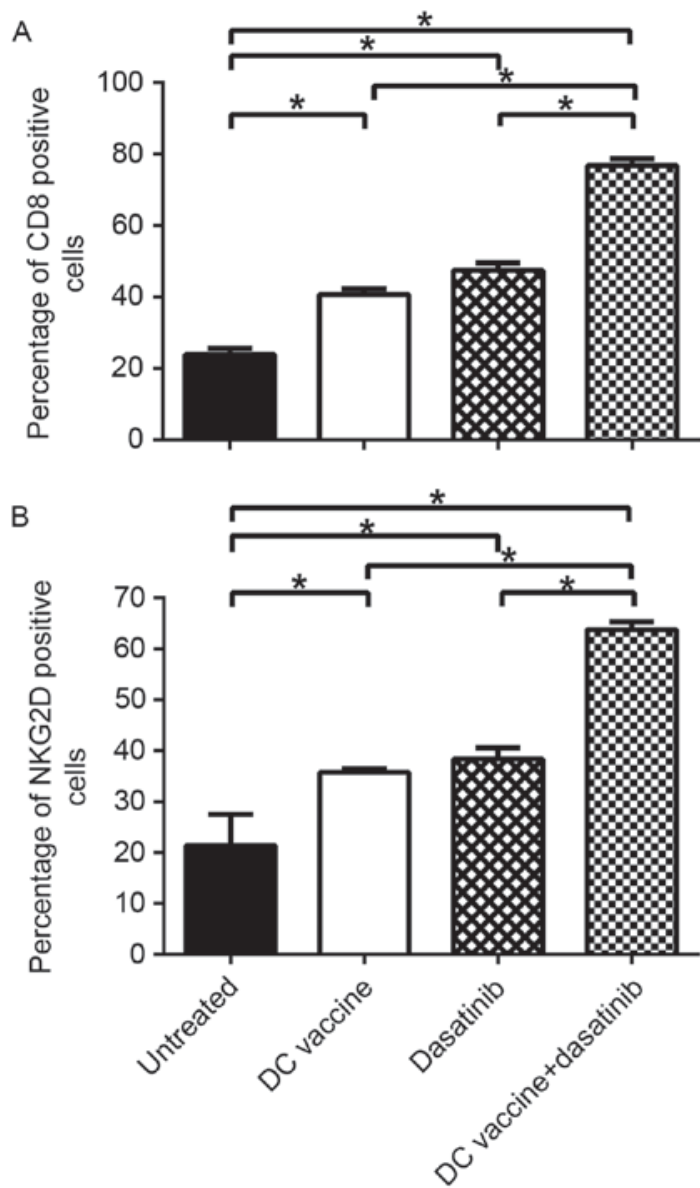

Figure 4. Alterations in the number of $\mathrm{CD}^{+} \mathrm{T}$ cells and NK cells subsequent to different treatments. $\mathrm{CD} 8^{+} \mathrm{T}$ cells and NK cells in each cohort were identified by standard immunohistochemistry and the ratio of (A) $\mathrm{CD}^{+} \mathrm{T}$ cells and (B) NK cells were examined in all tumors. ${ }^{*} \mathrm{P}<0.05$. CS, cluster of differentiation; NK, natural killer; DC, dendritic cell; NKG2D, natural-killer group 2, member D.

tumor sections was performed using CD31 as an indicator of angiogenesis. It was found that microvessel density was slightly decreased in the dasatinib alone or DC vaccine alone groups compared to the untreated control group. By contrast, the combined treatment group showed significantly reduced inhibition of intratumoral microvessel density compared to the other three groups (Fig. $2 \mathrm{H} ; \mathrm{P}<0.05$ ).

Dasatinib and DC vaccine inhibited the proliferation and enhanced the apoptosis of $4 T 1$ tumor cells synergistically. To assess the inhibition of proliferation and the induction of apoptosis after different treatments, immunohistochemistry of Ki-67, as a maker of proliferation, and TUNEL assay, as an indicator of apoptosis, were performed. DC vaccine group, dasatinib group and combination group exhibited decreased Ki-67 expression level related to untreated control group (Fig. 3A) $(\mathrm{P}<0.05)$. However, only the combined treatment exhibited a significantly increased inhibitory effect on proliferation compared to the untreated control. Furthermore, TUNEL assay showed that apoptotic cells were significantly increased in mice of combined treatment group compared to untreated control group or either single treatment group (Fig. 3B; $\mathrm{P}<0.05$ ). 
Combination of dasatinib and DC vaccine enhanced the expansion of $\mathrm{CD}^{+} \mathrm{T}$ cells and $\mathrm{NK}$ cells in the tumor microenvironment. Previous studies have indicated that the antitumor effect of dasatinib is largely dependent on the expansion of $\mathrm{CD}^{+} \mathrm{T}$ cells and NK cells. Subsequently, the presence of immune cells in the tumor microenvironment was measured by immunohistochemistry. Compared to untreated control mice, the ratio of $\mathrm{CD}^{+} \mathrm{T}$ cells in tumor tissues from the DC vaccine, dasatinib or combined treatment groups was significantly increased $(\mathrm{P}<0.05)$. Notably, in mice treated with dasatinib plus DC vaccine, the expression level of $\mathrm{CD}^{+} \mathrm{T}$ cells was significantly increased compared with the mice treated with dasatinib alone or DC vaccine alone (Fig. 4A; $\mathrm{P}<0.05$ ). Similarly, the ratio of NK cells was significantly increased in the combined treatment group compared with the other three groups (Fig. 4B; $\mathrm{P}<0.05$ ). Overall, these data indicate that the expansion of $\mathrm{CD}^{+} \mathrm{T}$ cell and $\mathrm{NK}$ cell at tumor site contributes to high anti-tumor efficacy of combination of dasatinib and DC vaccine.

\section{Discussion}

The rationale for combining dasatinib with DC vaccine in this study relied on the immunostimulatory off-target effects of dasatinib on several immune cells (7-11). In order to improve its potent immunostimulatory ability, dasatinib has been studied in combination with cytotoxic therapies, including chemotherapy, which may cause the release of tumor cell antigens, a crucial trigger for immune responses (21). However, cytotoxic therapies showed limited clinical efficacy, with potential increased frequency of adverse events (22). Immunotherapy is an ideal therapeutic adjuvant, as it is more effective in enhancing immune response and has low toxicity (23). Additionally, dasatinib combined with DC vaccine has resulted in marked effects in certain tumor models $(8,19)$. In the present study, 4T1 cell-induced breast cancer was used to demonstrate that the combination of dasatinib and DC vaccine significantly inhibits tumor growth and metastasis.

Recruitment of regulatory $\mathrm{T}$ cell (Tregs) and myeloid-derived suppressor cells (MDSCs) to the tumor microenvironment is one of the important mechanisms of immune tolerance $(24,25)$ and contributes to tumor initiation and progression $(26,27)$. Increased levels of MDSC and Tregs are associated with poor prognosis in multiple tumor types $(28,29)$. Previous studies in vitro showed dasatinib inhibits all subsets of T cells, without impairing its viability (30-33). However, previous studies have reported opposite immunostimulatory effects in vivo $(5,6,10,11,34)$. It is hypothesized that the inhibitory effect of dasatinib on suppressor cells, including Tregs and MDSCs, far outweigh the inhibition on other effector $\mathrm{T}$ cells, resulting in Treg and MDSC-mediated effector T cells activation and expansion $(35,36)$. Another possible explanation for this discrepancy is that in vitro culture may not completely reproduce in vivo conditions; the plasma half-life of dasatinib was reported to be only 3-4 h (37), and the transient inhibition on $\mathrm{T}$ cells could be reversed by drug removal $(31,37)$ in vivo. In addition, multiple preclinical and clinical studies showed that other TKIs, such as sunitinib $(38-42)$ and axitinib $(43,44)$, can decrease immunosuppressing MDSCs and Tregs in solid tumors via mechanisms involving c-kit, Stat3 and possibly
VEGF inhibition. Similar mechanisms on dasatinib are thought to be helpful to suppress Tregs and MDSCs $(8,19)$, but this possibility requires additional investigation.

The repertoire of chemokines in the tumor microenvironment is known to regulate the migration and infiltration of leukocytes via binding their various $G$ protein-coupled receptors. Dasatinib combined with the anti-X40 regimen decreases the ratio of Tregs to $\mathrm{CD}^{+}$effector $\mathrm{T}$ cells partly by upregulating CXCL9, CXCL10 and CXCL11 chemokines at the tumor site, resulting in substantially improved therapeutic efficacy compared to treatment with either single modality (19). Furthermore, the chemokine ligand/receptor binding would cause a positive feedback loop that attracts more effector $\mathrm{T}$ cells into the tumor site by producing more interferon (IFN)- $\gamma$, which further upregulates CXCL9, CXCL10 and CXCL11 $(45,46)$. Lowe et al (8) showed that treatment of mice inoculated with M05 melanoma cells with dasatinib and peptide-pulsed DC improved the recruitment of these chemokines to tumor microenvironment with increased secretion of IFN- $\gamma$ and activation and recruitment of Type-1, vaccine-induced $\mathrm{CXCR} 3^{+} / \mathrm{CD}^{+}$tumor-infiltrating lymphocytes into the tumor microenvironment.

DC-based immunotherapy is considered to effectively recognize and eradicate the malignant cell population, including intratumoral, peritumoral, distant and widely disseminated cancer cells. However, DC-based immune monotherapy has shown only limited transient response (47-49), which is in line with the present findings using DC vaccine alone. Previous studies suggest that dasatinib pretreatment could improve the tumor microenvironment to be ready for effective DC-based immunotherapy. Wolfl et al (17) observed that dasatinib significantly improved the production of IL-12p70 during TLR2/4-triggered DC activation. Nerreter et al (18) found that the number of migratory DCs in a dasatinib-pretreated LPS-matured DC cohort was significantly increased compared with the LPSonly-matured DC cohort.

The present study confirmed that the treatment protocol using dasatinib and DC vaccine was more effective in inhibiting 4T1 breast tumor growth and metastasis compared to either single therapy with dasatinib or DC vaccine. The synergistic therapeutic effect appeared to be mainly dependent on the expansion of CD8 ${ }^{+} \mathrm{T}$ cells and NK cells. Yang et al (19) reported that the superior therapeutic effect of dasatinib and anti-OX40 was largely due to $\mathrm{T}$ cell-mediated immunity, as $\mathrm{CD}^{+}{ }^{+}$or $\mathrm{CD} 8^{+} \mathrm{T}$-cell depletion experiment showed that reduced $\mathrm{CD}^{+}$or $\mathrm{CD}^{+} \mathrm{T}$ cells level lead to shorter survival time. Although the NK-cell-mediated innate immune response can mount antigen-independent anti-tumor responses and may be important to drive an effective immune response, the generation of tumor-specific CTL is considered essential for effective anti-tumor immunity.

In conclusion, the protocol using dasatinib with 4T1-antigen-loaded DC vaccine demonstrated synergistic antitumor efficacy on 4T1 breast cancer cell proliferation, apoptosis, metastasis and angiogenesis, with promising immunostimulatory effects for the expansion of $\mathrm{CD}^{+} \mathrm{T}$ cells and NK cells. Due to the unique ability of priming and boosting $\mathrm{T}$ cells and NK cells, DC should be mainly responsible for the achieved therapeutic effect. However, the addition of dasatinib leads to significant synergistic immunostimulatory effects. 
Therefore, dasatinib combined with DC vaccine is a possible modality for the effective treatment of metastatic breast cancer.

\section{Acknowledgements}

Not applicable.

\section{Funding}

No funding was received.

\section{Availability of data and materials}

The datasets generated and analyzed in the present study are included in this published article.

\section{Authors' contributions}

NS and XW conceived and designed the study. NS and HG performed the experiments and wrote the paper. NS, HG, JR and $\mathrm{SH}$ collected, analyzed and interpreted the data of the work. JR and SH reviewed and edited the manuscript. All authors read and approved the manuscript.

\section{Ethics and consent to participate}

All procedures involving animals were approved by the Ethics Committee of Tianjin Medical University. All animal studies also comply with the ARRIVE guidelines and the AVMA euthanasia guidelines 2013 .

\section{Consent for publication}

Not applicable.

\section{Competing interests}

The authors declare that they have no competing interests.

\section{References}

1. Balkwill F and Coussens LM: Cancer: An inflammatory link. Nature 431: 405-406, 2004.

2. Wang N, Reeves KJ, Brown HK, Fowles ACM, Docherty FE, Ottewell PD, Croucher PI, Holen I and Eaton CL: The frequency of osteolytic bone metastasis is determined by conditions of the soil, not the number of seeds; evidence from in vivo models of breast and prostate cancer. J Exp Clin Cancer Res 34: 124, 2015.

3. Larkin JR, Dickens AM, Claridge TD, Bristow C, Andreou K, Anthony DC and Sibson NR: Early diagnosis of brain metastases using a biofluids-metabolomics approach in mice. Theranostics 6 : 2161-2169, 2016.

4. Sun DW, Zhang HD, Mao L, Mao CF, Chen W, Cui M, Ma R, Cao HX, Jing CW, Wang Z, et al: Luteolin inhibits breast cancer development and progression in vitro and in vivo by suppressing notch signaling and regulating MiRNAs. Cell Physiol Biochem 37: 1693-1711, 2015.

5. Kreutzman A, Juvonen V, Kairisto V, Ekblom M, Stenke L, Seggewiss R, Porkka K and Mustjoki S: Mono/oligoclonal T and NK cells are common in chronic myeloid leukemia patients at diagnosis and expand during dasatinib therapy. Blood 116: 772-782, 2010.

6. Mustjoki S, Auvinen K, Kreutzman A, Rousselot P,Hernesniemi S, Melo T, Lahesmaa-Korpinen AM, Hautaniemi S, Bouchet S, Molimard M, et al: Rapid mobilization of cytotoxic lymphocytes induced by dasatinib therapy. Leukemia 27: 914-924, 2013.
7. Gridling M, Ficarro SB, Breitwieser FP, Song L, Parapatics K, Colinge J, Haura EB, Marto JA, Superti-Furga G, Bennett KL and Rix U: Identification of kinase inhibitor targets in the lung cancer microenvironment by chemical and phosphoproteomics. Mol Cancer Ther 13: 2751-2762, 2014.

8. Lowe DB, Bose A, Taylor JL, Tawbi H, Lin Y, Kirkwood JM and Storkus WJ: Dasatinib promotes the expansion of a therapeutically superior T-cell repertoire in response to dendritic cell vaccination against melanoma. Oncoimmunology 3: e27589, 2014.

9. Iriyama N, Fujisawa S, Yoshida C, Wakita H, Chiba S, Okamoto S, Kawakami K, Takezako N, Kumagai T, Inokuchi K, et al: Early cytotoxic lymphocyte expansion contributes to a deep molecular response to dasatinib in patients with newly diagnosed chronic myeloid leukemia in the chronic phase: Results of the D-first study. Am J Hematol 90: 819-824, 2015.

10. Shimura Y,Horiike S, Tsutsumi Y,Hatsuse M,Okano A,FuchidaS, Kobayashi T, Matsumoto Y, Kuroda J, Kawata-Iida E, et al: The longitudinal analysis of large granular lymphocytosis in patients with Philadelphia chromosome-positive leukemia treated with dasatinib. Int J Hematol 102: 426-433, 2015.

11. Christiansson L, Soderlund S, Mangsbo S, Hjorth-Hansen H, Hoglund M, Markevarn B, Richter J, Stenke L, Mustjoki S, Loskog A and Olsson-Strömberg U: The tyrosine kinase inhibitors imatinib and dasatinib reduce myeloid suppressor cells and release effector lymphocyte responses. Mol Cancer Ther 14: 1181-1191, 2015.

12. Kim DH, Kamel-Reid S, Chang H, Sutherland R, Jung CW, Kim HJ, Lee JJ and Lipton JH: Natural killer or natural killer/T cell lineage large granular lymphocytosis associated with dasatinib therapy for Philadelphia chromosome positive leukemia. Haematologica 94: 135-139, 2009.

13. Finn RS, Bengala C, Ibrahim N, Roche H, Sparano J, Strauss LC, Fairchild J, Sy O and Goldstein LJ: Dasatinib as a single agent in triple-negative breast cancer: Results of an open-label phase 2 study. Clin Cancer Res 17: 6905-6913, 2011.

14. Fornier MN, Morris PG, Abbruzzi A, D'Andrea G, Gilewski T, Bromberg J, Dang C, Dickler M, Modi S, Seidman AD, et al: A phase I study of dasatinib and weekly paclitaxel for metastatic breast cancer. Ann Oncol 22: 2575-2581, 2011.

15. Somlo G, Atzori F, Strauss LC, Geese WJ, Specht JM, Gradishar WJ, Rybicki A, Sy O, Vahdat LT and Cortes J: Dasatinib plus capecitabine for advanced breast cancer: Safety and efficacy in phase I study CA180004. Clin Cancer Res 19: 1884-1893, 2013.

16. Algazi AP, Weber JS, Andrews SC, Urbas P, Munster PN, DeConti RC, Hwang J, Sondak VK, Messina JL, McCalmont T and Daud AI: Phase I clinical trial of the Src inhibitor dasatinib with dacarbazine in metastatic melanoma. Br J Cancer 106: 85-91, 2012.

17. Wolfl M, Schwinn S, Yoo YE, Ress ML, Braun M, Chopra M, Schreiber SC, Ayala VI, Ohlen C, Eyrich M, et al: Src-kinase inhibitors sensitize human cells of myeloid origin to Toll-like-receptor-induced interleukin 12 synthesis. Blood 122: 1203-1213, 2013.

18. Nerreter T, Köchel C, Jesper D, Eichelbrönner I, Putz E, Einsele H and Seggewiss-Bernhardt R: Dasatinib enhances migration of monocyte-derived dendritic cells by reducing phosphorylation of inhibitory immune receptors Siglec-9 and Siglec-3. Exp Hematol 42: 773-782.e1-3, 2014.

19. Yang Y, Liu C, Peng W, Lizee G, Overwijk WW, Liu Y, Woodman SE and Hwu P: Antitumor T-cell responses contribute to the effects of dasatinib on c-KIT mutant murine mastocytoma and are potentiated by anti-OX40. Blood 120: 4533-4543, 2012.

20. Weidner N: Current pathologic methods for measuring intratumoral microvessel density within breast carcinoma and other solid tumors. Breast Cancer Res Treat 36: 169-180, 1995.

21. Lee F, Jure-Kunkel MN and Salvati ME: Synergistic activity of ixabepilone plus other anticancer agents: Preclinical and clinical evidence. Ther Adv Med Oncol 3: 11-25, 2011.

22. Somlo G, Atzori F, Strauss LC, Geese WJ, Specht JM, Gradishar WJ, Rybicki A, Sy O, Vahdat LT and Cortes J: Dasatinib plus capecitabine for advanced breast cancer: Safety and efficacy in phase I study CA180004. Clin Cancer Res 19: 1884-93, 2013.

23. Chen J,Zhao Y, Chu X, Lu Y, Wang S and Yi Q: Dectin-1-activated dendritic cells: A potent Th9 cell inducer for tumor immunotherapy. Oncoimmunology 5: e1238558, 2016.

24. Schietinger A and Greenberg PD: Tolerance and exhaustion: Defining mechanisms of T cell dysfunction. Trends Immunol 35: 51-60, 2014. 
25. Makkouk A and Weiner GJ: Cancer immunotherapy and breaking immune tolerance: New approaches to an old challenge. Cancer Res 75: 5-10, 2015.

26. Garcia AJ, Ruscetti M, Arenzana TL, Tran LM, Bianci-Frias D, Sybert E, Priceman SJ, Wu L, Nelson PS, Smale ST and Wu H: Pten null prostate epithelium promotes localized myeloid-derived suppressor cell expansion and immune suppression during tumor initiation and progression. Mol Cell Biol 34: 2017-2028, 2014.

27. Keskinov AA and Shurin MR: Myeloid regulatory cells in tumor spreading and metastasis. Immunobiology 220: 236-242, 2015.

28. Almand B, Clark JI, Nikitina E, van Beynen J, English NR, Knight SC, Carbone DP and Gabrilovich DI: Increased production of immature myeloid cells in cancer patients: A mechanism of immunosuppression in cancer. J Immunol 166: 678-689, 2001.

29. Almand B, Resser JR, Lindman B, Nadaf S, Clark JI, Kwon ED, Carbone DP and Gabrilovich DI: Clinical significance of defective dendritic cell differentiation in cancer. Clin Cancer Res 6: 1755-1766, 2000.

30. Blake S, Hughes TP, Mayrhofer G and Lyons AB: The Src/ABL kinase inhibitor dasatinib (BMS-354825) inhibits function of normal human T-lymphocytes in vitro. Clin Immunol 127: 330-339, 2008.

31. Blake SJ, Bruce LA, Fraser CK, Hayball JD and Hughes TP: Dasatinib suppresses in vitro natural killer cell cytotoxicity. Blood 111: 4415-4416, 2008.

32. Schade AE, Schieven GL, Townsend R, Jankowska AM, Susulic V, Zhang R, Szpurka H and Maciejewski JP: Dasatinib, a small-molecule protein tyrosine kinase inhibitor, inhibits T-cell activation and proliferation. Blood 111: 1366-1377, 2008.

33. Weichsel R, Dix C, Wooldridge L, Clement M, Fenton-May A, Sewell AK, Zezula J, Greiner E, Gostick E, Price DA, et al Profound inhibition of antigen-specific T-cell effector functions by dasatinib. Clin Cancer Res 14: 2484-2491, 2008.

34. Valent JN and Schiffer CA: Prevalence of large granular lymphocytosis in patients with chronic myelogenous leukemia (CML) treated with dasatinib. Leuk Res 35: e1-e3, 2011

35. Yang L, DeBusk LM, Fukuda K, Fingleton B, Green-Jarvis B, Shyr Y, Matrisian LM, Carbone DP and Lin PC: Expansion of myeloid immune suppressor $\mathrm{Gr}+\mathrm{CD} 1 \mathrm{~b}+$ cells in tumor-bearing host directly promotes tumor angiogenesis. Cancer Cell 6 : 409-421, 2004.

36. Heine A, Held SA, Bringmann A, Holderried TA and Brossart P: Immunomodulatory effects of anti-angiogenic drugs. Leukemia 25: 899-905, 2011.

37. Weisberg E, Manley PW, Cowan-Jacob SW, Hochhaus A and Griffin JD: Second generation inhibitors of BCR-ABL for the treatment of imatinib-resistant chronic myeloid leukaemia. Nat Rev Cancer 7: 345-356, 2007.

38. Finke JH, Rini B, Ireland J, Rayman P, Richmond A, Golshayan A, Wood L, Elson P, Garcia J, Dreicer R and Bukowski R: Sunitinib reverses type-1 immune suppression and decreases T-regulatory cells in renal cell carcinoma patients. Clin Cancer Res 14: 6674-6682, 2008.

39. Ko JS, Zea AH, Rini BI, Ireland JL, Elson $P$, Cohen $P$, Golshayan A, Rayman PA, Wood L, Garcia J, et al: Sunitinib mediates reversal of myeloid-derived suppressor cell accumulation in renal cell carcinoma patients. Clin Cancer Res 15: 2148-2157, 2009
40. Ozao-Choy J, Ma G, Kao J, Wang GX, Meseck M, Sung M, Schwartz M, Divino CM, Pan PY and Chen SH: The novel role of tyrosine kinase inhibitor in the reversal of immune suppression and modulation of tumor microenvironment for immune-based cancer therapies. Cancer Res 69: 2514-2522, 2009.

41. Xin H, Zhang C, Herrmann A, Du Y, Figlin R and $\mathrm{Yu} \mathrm{H}$ : Sunitinib inhibition of Stat 3 induces renal cell carcinoma tumor cell apoptosis and reduces immunosuppressive cells. Cancer Res 69: 2506-2513, 2009.

42. Bose A, Taylor JL, Alber S, Watkins SC, Garcia JA, Rini BI, Ko JS, Cohen PA, Finke JH and Storkus WJ: Sunitinib facilitates the activation and recruitment of therapeutic anti-tumor immunity in concert with specific vaccination. Int J Cancer 129: 2158-2170, 2011.

43. Bose A, Lowe DB, Rao A and Storkus WJ: Combined vaccine+axitinib therapy yields superior antitumor efficacy in a murine melanoma model. Melanoma Res 22: 236-243, 2012

44. YuanH,CaiP,LiQ,Wang W,Sun Y,XuQandGu Y: Axitinibaugments antitumor activity in renal cell carcinoma via STAT3-dependent reversal of myeloid-derived suppressor cell accumulation. Biomed Pharmacother 68: 751-756, 2014.

45. Bonecchi R, Bianchi G, Bordignon PP, D'Ambrosio D, Lang R, Borsatti A, Sozzani S, Allavena P, Gray PA, Mantovani A and Sinigaglia F: Differential expression of chemokine receptors and chemotactic responsiveness of type $1 \mathrm{~T}$ helper cells (Th1s) and Th2s. J Exp Med 187: 129-134, 1998.

46. Basset L, Chevalier S, Danger Y, Arshad MI, Piquet-Pellorce C, Gascan $\mathrm{H}$ and Samson M: Interleukin-27 and IFNg regulate the expression of CXCL9, CXCL10, and CXCL11 in hepatitis. J Mol Med (Berl) 93: 1355-1367, 2015.

47. Bol KF, Figdor CG, Aarntzen EH, Welzen ME, van Rossum MM, Blokx WA, van de Rakt MW, Scharenborg NM, de Boer AJ, Pots JM, et al: Intranodal vaccination with mRNAoptimized dendritic cells in metastatic melanoma patients. OncoImmunology 4: e1019197, 2015.

48. Carreno BM, Magrini V, Becker-Hapak M, Kaabinejadian S, Hundal J, Petti AA, Ly A, Lie WR, Hildebrand WH, Mardis ER and Linette GP: Cancer immunotherapy. A dendritic cell vaccine increases the breadth and diversity of melanoma neoantigen-specific T cells. Science 348: 803-808, 2015.

49. Pizzurro GA and Barrio MM: Dendritic cell-based vaccine efficacy: Aiming for hot spots. Front Immunol 6: 91, 2015.

This work is licensed under a Creative Commons

Attribution-NonCommercial-NoDerivatives 4.0 International (CC BY-NC-ND 4.0) License. 